\title{
Influence of SPD on Whiteness value of FWA treated samples
}

\begin{abstract}
The Whiteness of textiles, plastics, paper and paints is important aspects, which are considered in daily life. Customers usually prefer white products, especially in summer because of comfort reasons. In addition, white, being an achromatic color has a physiological effect on human sensation. Generally, High whiteness is not possible to get through only bleaching process. Therefore, the application of FWA's (Fluorescence Whitening Agents) is a common practice in industry where the need to achieve "high" whiteness. In the case of white materials, the nature of these chemicals is to absorb light in the invisible, or near ultra-violet, region of the spectrum and then re-emit this light as fluorescence in the visible region of the spectrum. This re-emitted light generally occurs between 420-500nm. The effect is a greater degree of reflectance in the blue region of the spectrum, therefore a "bluer" white. In effect, this process produced much whiter whites! Consequently to other color attributes of an object depends on many factors, such as lighting (illumination), size of sample, and background and surrounding colors. Much more importantly, color is a subjective phenomenon and depends on the observer. In this article were discussed the influence of SPD (Spectral Power Distribution) tested light sources on whiteness. Solid-state lighting (white LEDs, etc.) is necessary to improve by a full-visible-spectrum technology because standard white LEDs are unable to activate FWA's sufficiently. Ra does not capture this issue or other conventional color rendition metrics, although it corresponds to a very large color distortion for shades of white. Full-visible-spectrum LEDs emit no harmful ultra-violet light, but enough violet light to properly excite FWA's and render whites like natural sources.
\end{abstract}

Keywords: Colorimetry, whiteness, spectral power distribution, LED.

\section{Introduction}

Whiteness is an attribute of colors of high luminous reflectance and low purity situated in a relatively narrow region of the color space along dominant wavelengths between $570 \mathrm{~nm}$ and $470 \mathrm{~nm}$. Whiteness of paper as well as textiles is a commercially important property, generation of suitable white materials is important to satisfy high lightness and desired chromatic requirements from an aesthetic perspective and to provide a suitable base for subsequent coloration via dyeing and printing [1, 2]. However, most natural and synthetic fibers contain colorants that detract from their apparent whiteness [3-6]. Two common approaches used to improve the whiteness of paper and textile materials are chemical bleaching and fluorescent (optical) whitening

In chemical bleaching, oxidants or reducing agents are used to eliminate the colored impurities in fibers and it improves the whiteness. However, these methods may not be commercially acceptable where the finished product is expected to be a bright white. Fluorescing whitening agents (FWA) emitting in the blue region of the electromagnetic spectrum are especially used to enhance the brightness and whiteness of paper and textile materials.

According to Ganz [7], the assessment of whiteness depends on individual preference, the level and spectral power distribution of the sample irradiation, the color of the surround, and the acquired preconceptions in various trades. Despite of the problems presented by the colorimetry of fluorescent samples, most observers are able to arrange white samples of different luminous reflectance, hue and saturation in a one-dimensional order according to whiteness, although little general agreement on whiteness can be reached. Regardless of this disparity, attempts have been made to elaborate a standardized whiteness formula for commercial whites. The general agreement is that a sample is perceived as whiter, lighter and bluer. Thus, whiteness are characterized by high levels of luminosity and finite saturation, with a blue hue [8].

Several whiteness formulas are based on the object's chromaticity, which shift towards the blue direction and follow a linear form $[9,10]$ :

(1) $W=Y-\omega \cos (\eta+\varphi) / \cos (\varphi)\left(x-x_{0}\right)-\omega \sin (\eta+\varphi) / \cos (\varphi)\left(y-y_{0}\right)$ where $Y$ is the tristimulus value $Y$ of the sample, i.e. its luminance factor ( $Y=100$ for a perfect diffuser); $\omega$ is the sensitivity of whiteness to saturation (e.g. to a color shift along $\left.\lambda_{d} \sim 470 \mathrm{~nm}\right) ; \eta$ is the angle between the $x$-axis and the direction of $\lambda_{d}$, i.e. $\tan (\eta)=\left(y_{0}-y_{d}\right) /\left(x_{0}-x_{d}\right) ; \varphi$ is a small angle which represents tint preference (e.g., the fact that maximal whiteness enhancement is not exactly in the direction of $\left.\lambda_{d}\right) ;\left(x_{0}, y_{0}\right)$ are the chromaticity coordinates of the reference illuminant; $\left(x_{d}, y_{d}\right)$ are the chromaticity coordinates the dominant wavelength $\lambda_{d}$; and $(x, y)$ are the coordinates of the object under consideration. All these quantities pertain to a specific color space and the corresponding Color-Matching Functions (CMFs). The values of $\omega$ and $\varphi$ are derived from experimental data [11].

One of the most widely used whiteness quantities, the CIE whiteness is a particular solution of equation (1). CIE whiteness is defined as follows for the D65 Illuminant and $10^{\circ}$ observer $\left(\mathrm{D} 65 / 10^{\circ}\right)[12]$ :

$$
W=Y+800\left(x_{0}-x\right)+1700\left(y_{0}-y\right)
$$

David et al [11] proposed general form of CIE whiteness, which can be expressed as:

$$
W=Y-P\left(x-x_{0}\right)-Q\left(y-y_{0}\right)
$$

where values of $P$ and $Q$ can be derived for any CCT (Correlated Color Temperature) between 2000 and $7000 \mathrm{~K}$ taking into account the chromaticity coordinates of the source.

When determining the surface color of a solid opaque sample material, the most important object related property is the total radiance factor $\beta_{T}(\lambda)$ of the material, which defines the spectral composition of the light reflected and emitted by the sample. The total radiance factor is a sum of the reflection radiance factor $\beta_{R}(\lambda)$ and the fluorescence radiance factor $\beta_{L}(\lambda)$, the latter being zero for all nonfluorescent materials [13]:

$$
\beta_{T}=\beta_{R}+\beta_{L}
$$

The reflection radiance factor is not sensitive to specific illumination conditions, but the fluorescence radiance factor 
for a fluorescent material depends critically on the illumination. The actual light sources in practical measurement instruments differ from the standard illuminants. However, the fluorescence radiance factor can be derived for a specific illuminant from sample specific illumination independent bi-spectral luminescent radiance factor data. Additionally, measured spectral reflectance is not a standard fixed quantity [14]. It is influenced by a variety of factors such as the geometry of the measurement device, aperture, light source, filters and measurement set up, which all influence the data that are acquired from an instrument. These factors may differ from one instrument to another. Thus, comparing data from at least two different instruments could be an appropriate approach to enable mills and their customers to have more accurate and reliable data for optical analyses of materials that contain dye or FWA. Nevertheless Griesser showed in his work [9] that, along with adjustment of sample illumination to a given standard to standardize the whiteness values of the different measurement instrument constructions and illuminations, matching formula parameters are greatly improves the whiteness assessments.

The purpose of this present work is to qualitatively study, the impact of the SPD (spectral power distribution) on the illumination in the fluorescence absorption band of FWA on the measured whiteness value.

\section{Materials and methods}

We consider how various sources render whiteness for a series of calibrated whiteness standards. A total of 6 plastic samples of the CIBA whiteness scale was measured in order of whiteness when viewed on a pale gray background under four white light sources, from which two approximating the CIE D65 illuminant. Tristimulus values of these samples are presented in Table 1:

Table 1. The tristimulus values of the tested sample (D65/10)

\begin{tabular}{|c|c|c|c|}
\hline Sample & $\mathrm{X}$ & $\mathrm{Y}$ & Z \\
\hline 2 & 81.68 & 86.47 & 82.93 \\
\hline 4 & 83.59 & 88.74 & 90.23 \\
\hline 6 & 84.18 & 89.00 & 96.49 \\
\hline 8 & 85.39 & 89.68 & 102.44 \\
\hline 10 & 85.53 & 89.17 & 107.52 \\
\hline 12 & 87.78 & 91.36 & 113.82 \\
\hline
\end{tabular}

The tested light sources were installed in viewing boxes together with UV fluorescent tube, simulating portion of UV radiation in daylight.

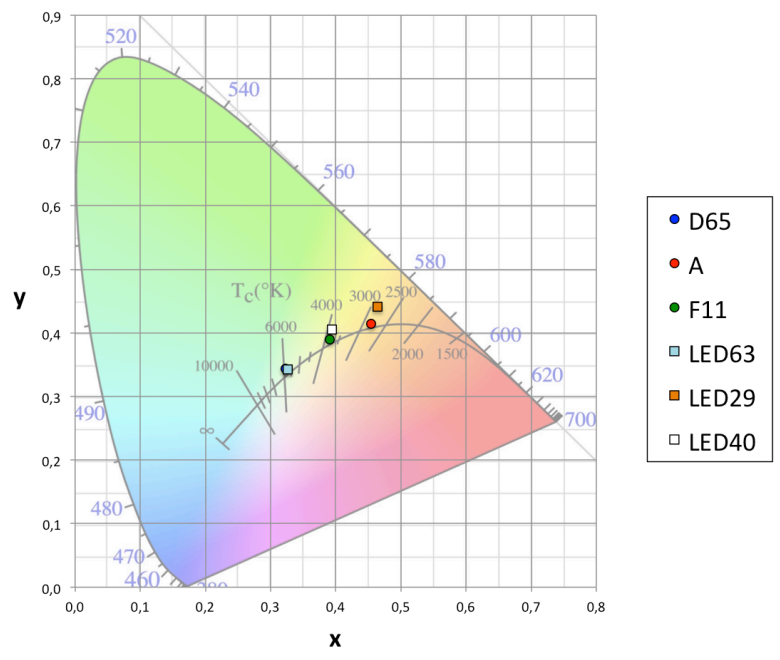

Fig.1. Chromaticity coordinates of tested light sources including illuminants $A$ and $F 11$ in $\mathrm{CIE} x, y$ diagram
The LED light sources we consider were typical bluepumped phosphor white-emitting LED and have a CCT of 2918 K (LED29) till $6338 \mathrm{~K}$ (LED63) as shown Table 2. The SPL III D65 is a professional daylight simulator based on the filtered tungsten lamp. The chromacity coordinates of these light sources are presented in the graph in Figure 1.

The chromacity coordinates of light source LED63 show a good approximation of daylight D65 in point of view of CCT, nevertheless its SPD documents known gap of energy on the beginning of visual part of electromagnetic spectrum together with gap on $485 \mathrm{~nm}$. Such problems are particularly solved by replacement of blue LED chip by violet one, following two phosphors technology $[15,16]$. Unfortunately the authors have any such light source for disposal during presented experiment.

Table 2. The parameters of the tested light sources

\begin{tabular}{|c|c|c|c|}
\hline Light source & CCT & CRI Ra & CQS \\
\hline LED6338 & $6338 \mathrm{~K}$ & 68.7 & 68.6 \\
\hline LED2918 & $2918 \mathrm{~K}$ & 59.8 & 64.0 \\
\hline LED4011 & $4011 \mathrm{~K}$ & 64.8 & 67.7 \\
\hline SPL III D65 & $6297 \mathrm{~K}$ & 94.7 & 94.7 \\
\hline
\end{tabular}

The spectral radiance of these light sources was measured by OceanOptics USB2000 spectrometer, which was connected with $30 \mathrm{~mm}$ diameter integrating sphere by optical fiber. Whole spectroradiometer was standardized by NIST calibrated tungsten light source. Measured data were collected by Ocean View v. 1.4.1 software and transferred in resulting SPD from LCAM color software. SPDs of tested light sources are shown on the graph in Figure 2:

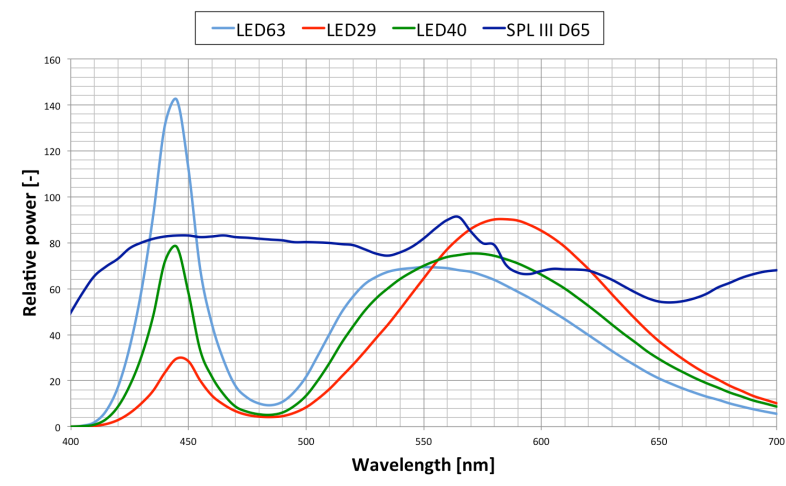

Fig.2. SPD of tested light sources

The spectral reflectance of each of the white sample was measured using the Spectraflash SF600plus (Datacolor Int., USA) reflectance spectrophotometer. In order to determine the appropriate whiteness values the spectrophotometer was calibrated for measuring the reflectance spectra and whiteness using, Hohensteiner Institute, Germany that offers a set of four textile samples with certified Ganz and CIE whiteness values for setting UV levels to $D_{65}$ conditions. These are four textile samples with the first one non-fluorescent, and the other three with increasing amounts of fluorescence. Since only whiteness data are provided, an external system defining reflectance levels must be used to complete the calibration system.

The CIE whiteness of the samples, measured by the SF600plus reflectance spectrophotometer at de: $8^{\circ}$ viewing conditions, varies between 54 and 140 (outdoors whiteness, i.e. D65 illumination). The two samples with the lowest whiteness contain no FWA, while the other samples contain an increasing amount of FWA.

Tristimulus values of CIBA plastic samples under tested light sources were measured by using of colorimeter Minolta CA2 10 at $45^{\circ} \mathrm{x}: 0^{\circ}$ viewing condition. 


\section{Results and discussion}

Figure 3 shows the plot of the CIE whiteness index selected 6 plastic samples of the CIBA whiteness scale measured by spectrophotometer against CIE whiteness index computed from tristimulus values measured by colorimeter Minolta CA210 under tested light sources without UV. It is visible that measured whiteness is low and starting from third sample almost constant.

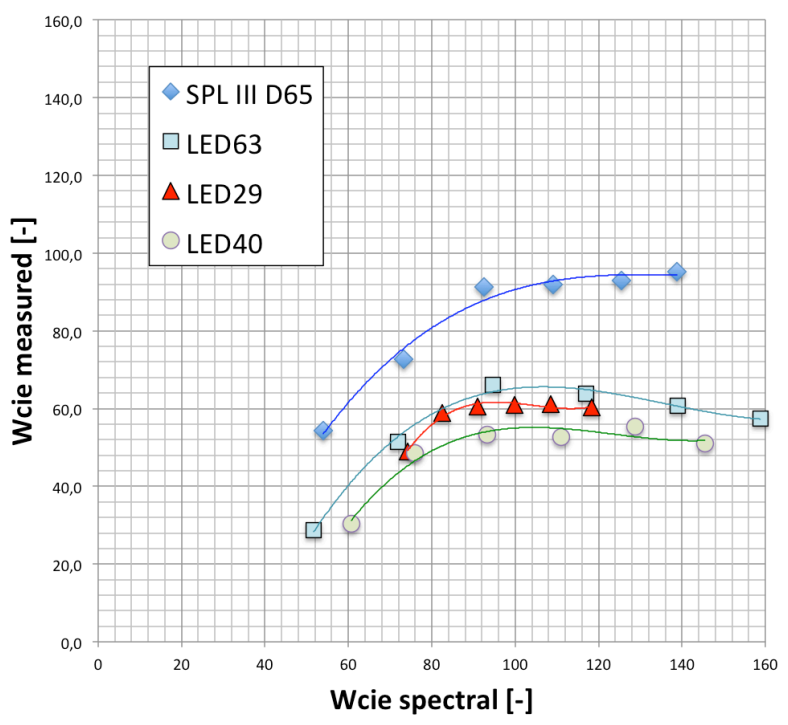

Fig.3. Relationship between CIE whiteness index measured by spectrophotometer (Wcie spectral) and CIE whiteness index measured by colorimeter under tested light sources without additional UV fluorescent tube.

From the graph in Figure 3 is evident, that all measured whiteness values are below 100 units. Such result confirms equation (4), which explain resulting total radiance factor as sum of two radiance factors (reflectance and fluorescence). The whiteness formulas described by equations (1), (2) and (3) are for ideal white equal to 100 . In case of real nonfluorescent samples whiteness indices will be below 100 . The real fluorescent samples illuminated by UV less radiation will appear as non-fluorescent samples, because FWA will be inactive. Such effect is documented by the graph in Figure 4, where is visible decreasing of reflectance, total radiance factor $\beta_{T}(\lambda)$ respectively. It is visible, that filtration of light below $420 \mathrm{~nm}$ will caused minimization of fluorescence radiance factor $\beta_{L}(\lambda) \rightarrow 0$.

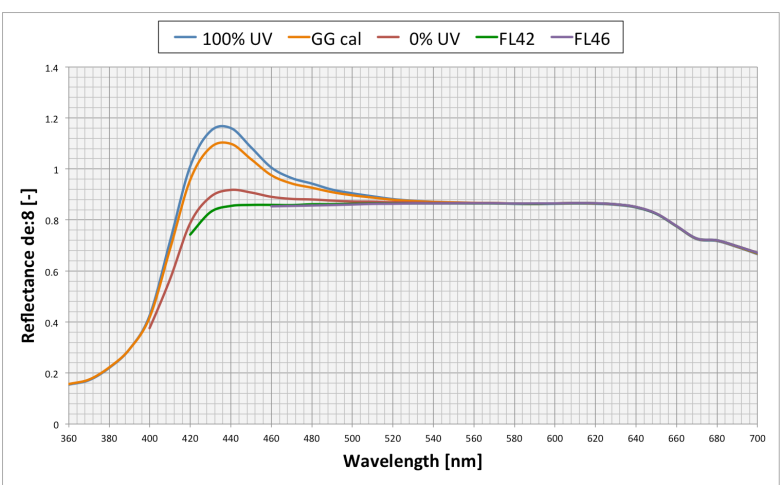

Fig.4. Reflectance curves of Sample 10 under different light conditions $(100 \%$ is full energy of xenon flash lamp, GG cal is calibrated amount of UV radiation following D65 standard, 0\% UV is light of lamp filtered by FL40 cut-off filter - radiation below $400 \mathrm{~nm}$ is absorbed by filter, FL42 - cut-off filter at $420 \mathrm{~nm}$ and FL46 - cut off filter at 460nm
This effect is also documented by lower CIE whiteness indices measured under LEDs in comparison to daylight simulator D65 from SpectraLight III (X-Rite) viewing cabinet, which is based on filtered tungsten. Exactly SPD of this daylight simulator contains low amount of UV radiation. Contrary to LEDs spectral power distribution of SPL III D65 simulator has any gaps in the visual part of spectrum.

Presented relations on plot in Figure 5 shows that only mentioned absence of emitted energy below $420 \mathrm{~nm}$ (Figure 2), which caused decreasing of the measured white under LEDs is not only one factor affecting resulting whiteness.

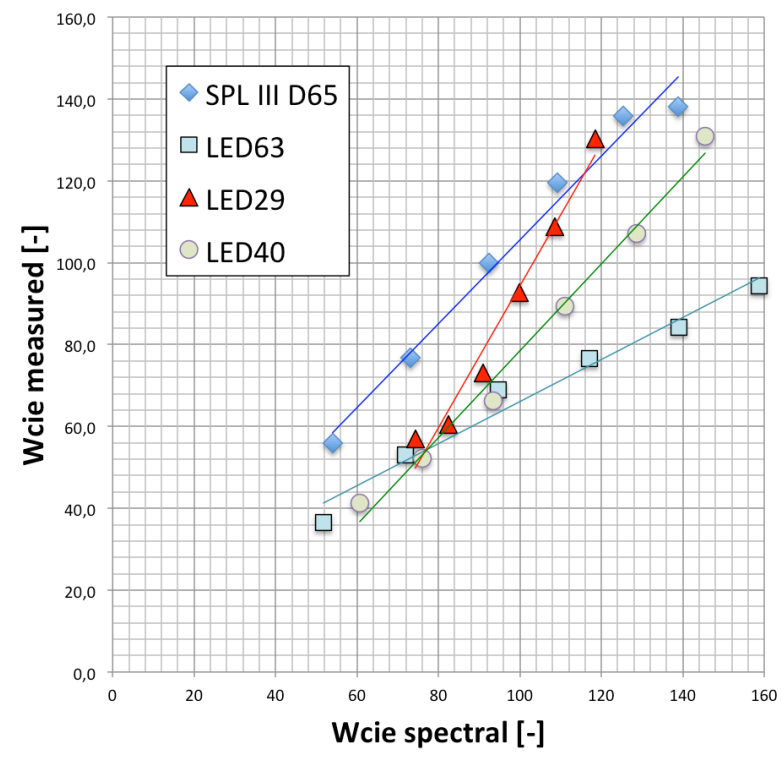

Fig.5. Relationship between CIE whiteness index measured by spectrophotometer (Wcie spectral) and CIE whiteness index measured by colorimeter under tested light sources with additional UV fluorescent tube.

It is shown that linear relation between measured data of CIE whiteness under SPL III daylight simulator conforms to data measured by spectrophotometer. Similar situation was found to LED light sources. The warm white LED (LED29) appear as more effective in point of view of slope of linear regression contrary to cool white LED (LED63), than neutral white is almost near to slope of SPL III daylight simulator. Such result is interesting, because the purpose of this work was a study of the impact of the SPD (spectral power distribution) in the fluorescence absorption band of FWA on the measured whiteness value and Figure 5 documents that resulting CIE whiteness for LED63 is less than 100 units, contrary to our expectations. Generally whiteness of assessed samples under a light source with higher CCT will appear higher in comparison to light sources with lower CCT.

Table 3. Difference in whiteness indices between Wcie (Eq.2) and proposed general form of CIE whiteness (Eq. 3)

\begin{tabular}{|c|c|c|c|}
\hline Sample & LED63 & LED29 & LED40 \\
\hline 2 & 0.2 & 0.1 & 0.2 \\
\hline 4 & 0.2 & 0.7 & 0.4 \\
\hline 6 & 0.2 & 1.2 & 0.6 \\
\hline 8 & 0.2 & 1.3 & 0.7 \\
\hline 10 & 0.2 & 1.9 & 0.9 \\
\hline 12 & 0.2 & 2.4 & 0.9 \\
\hline
\end{tabular}

Based on David et al [11] proposed general form of CIE whiteness we try to test the difference between whiteness indices computed following equations (2) and (3). Results presented in form of Table 3 shows that between both 
whiteness indices is relatively small difference, which slightly increased with whiteness index. Greater difference is between whiteness indices computed for LED29 because there is highest effect of shift on line of constant correlated color temperature as visible on graph in Figure 1 and documented in Table 4 by difference in CIE $u, v$ diagram $(\Delta u v)$. It is evident that difference between position of blackbody radiator and tested light source at the same temperature in chromacity diagram is approximately 20times higher for LED29 in comparison to LED63.

Table 4. Colorimetric and photometric parameters of tested light sources, including $P$ an $Q$ parameters used in Eq. 3

\begin{tabular}{|c|c|c|c|c|}
\hline Parameter & LED63 & LED29 & LED40 & SPLIII D65 \\
\hline P & 821 & 971 & 885 & 820 \\
\hline Q & 1686 & 1615 & 1657 & 1686 \\
\hline CCT & 6338 & 2919 & 4012 & 6298 \\
\hline CRv & 0,0005 & 0,0094 & 0,0066 & 0,0035 \\
\hline R(9-12) & 68,7 & 59,8 & 64,8 & 94,7 \\
\hline R9 & 28,0 & 5,6 & 17,3 & 88,7 \\
\hline LER(Im/W) & $-28,2$ & $-67,2$ & $-46,3$ & 77,1 \\
\hline CQS & 624,3 & 391,6 & 367,2 & 246,5 \\
\hline
\end{tabular}

In both tested equations (Eq. 2 and 3 ) are used beside tristimulus value $Y$ differences between chromaticity coordinates of the perfect diffuser $\left(x_{0}, y_{0}\right)$ and chromacity coordinates of measured samples $(x, y)$ under tested illuminant. Based on that, we try to analyze the changes of chromacity coordinates in CIE chromacity diagrams caused differences in the spectral power distribution of used light sources. Graphs in Figure 6 show chromacity coordinates selected samples FWA less sample and graphs in Figure 7 show sample with higher FWA concentration. Values marked as SPEC is chromacity coordinates calculated by using of tested light source SPD measured by spectroradiometer, -UV are chromacity coordinates measured by colorimeter under tested light source without UV radiation and +UV means chromacity coordinates measured by colorimeter under tested light source with UV radiation. It is evident that FWA less sample is insensitive on presence of UV radiation, contrary to sample with highest FWA concentration. Simultaneously, it is visible that difference between both samples under tested light source without UV radiation is only slightly shifted. That means, inactive FWA doesn't change chromacity of presented samples.
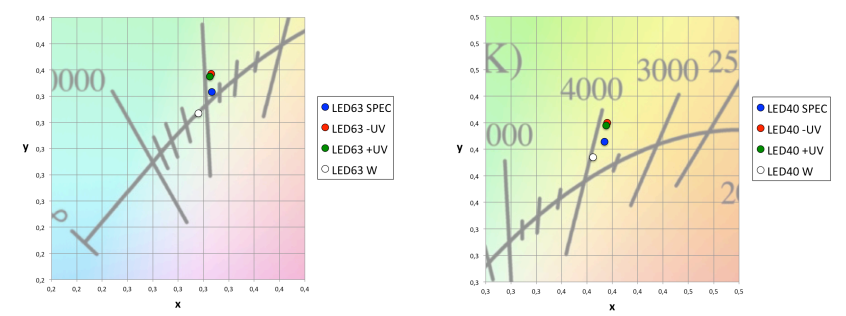

Fig.6. Chromaticity coordinates of FWA less sample 2 under LED63 (left) and LED40 (right)
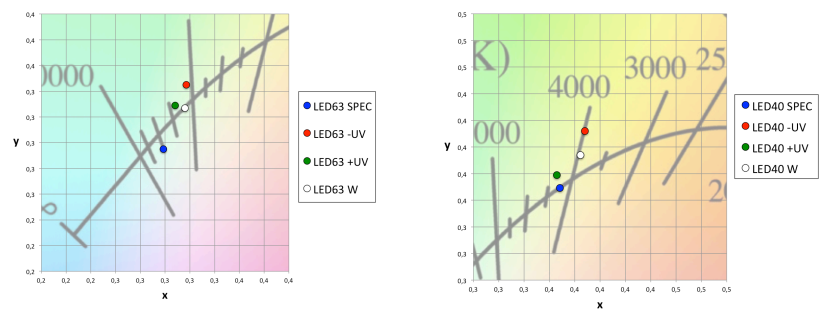

Fig.7. Chromaticity coordinates of sample ${ }^{\star} 12$ with maximal concentration of FWA under LED63 (left) and LED40 (right)
Graphs in Figure 7 document difference in chromacity shift caused by presence of UV radiation between LED63 and LED40 light source. Due to confirmation of this phenomenon was made study of whiteness scale dependence on chromacity difference calculated by equation (5):

$$
\Delta x y=\sqrt{\left(x-x_{0}\right)^{2}+\left(y-y_{0}\right)^{2}}
$$

Obtained results are presented by plot in Figure 8, where are visible two linear relations, which are sensitive on size of chromacity difference. It is visible that decreasing of measured whiteness caused by increasing of chromacity difference for whiteness values below 90 units. Whiteness values above 90 units are increased by same slope with increasing of chromacity difference. Such character of plot is of coarse caused by positive character of equation (5), if we will use orientation of chromacity difference as parameter following general form of whiteness formula in equation (1) result will be confirmation of this linear form.

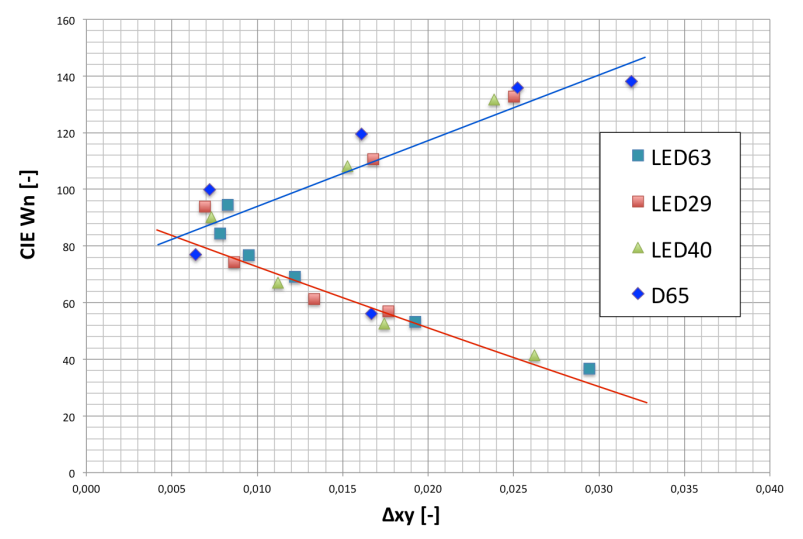

Fig.8. Relationship between CIE whiteness index (Eq.3) and chromacity differences of measured samples under UV containing irradiation by tested light sources

This analysis confirms usability of equation (3) for blue pumped LEDs in CCT interval <3000K; $7500 \mathrm{~K}>$. Therefore, we can speculate that low whiteness values of tested FWA containing samples under LED63 light source are caused by triplet excitation. The so-called triplet effect is an artifact, which is observed in whiteness and color assessments using instruments with pulsed light sources. With instruments having a continuous sample illumination the triplet effect is obviously not measurable. In absorption processes caused by continuous lamps or daylight interaction of light with species (molecules) in their shortlived singlet states are observed. With flash lamps enough long-lived triplet species can be produced. As a consequence also transitions between triplet states can be detected. This effect is mainly observed with certain FWA. With FWA additional absorption typically occurs in the wavelength region between 450 and $600 \mathrm{~nm}$, due this absorption as result is visible decreasing of luminosity. In case of blue pumped white LEDs we can see emission peak of blue LED at the wavelength $445 \mathrm{~nm}$ as visible on graph in Figure 2, which is near to fluorescence peak $432 \mathrm{~nm}$ of tested samples contains FWA as documented on graph in Figure 4. The SPDs in Figure 2 show that relative intensity of LED63 is at wavelength $445 \mathrm{~nm}$ twice times higher than LED40 intensity and more than four times higher than LED29. A combination of two emission bands (UV $-365 \mathrm{~nm}$ and blue $-445 \mathrm{~nm}$ ) is probably source of over excitation of FWA and resulting triplet effect. 


\section{Conclusion}

The appearance of fluorescing media does not only depend on the amount of radiation in the excitation band of the fluorophore but also on the SPD of the illumination in the emission band. A strong blue peak in the illumination can e.g. reflectance factor inhibit the effect of fluorescing whitening agents. This effect will impact the appearance of fluorescing media in all illuminations with large SPD variation such as LED illuminations.

\section{REFERENCES}

[1] J. Lin, R. Shamey, D. Hinks: Factors affecting the whiteness of optically brightened material, J. Opt. Soc. Am. A, 11, (29), 2012, $2289-2299$

[2] Vik M., Vikova M., Colour-appearance phenomena metamerism, Vlakna a Textil, Vol. 7, 2,2000,126-127

[3] R. H. Peters, Textile Chemistry, Vol. I: The Chemistry of Fibres (Elsevier, 1963).

[4] R. H. Peters, Textile Chemistry, Vol. II: Purification of Fibers (Elsevier, 1967).

[5] R. H. Peters, Textile Chemistry, Vol. III: The Physical Chemistry of Dyeing (Elsevier, 1975).

[6] M. Lewin, Handbook of Fiber Chemistry, 3rd ed. (CRC Press, 2007).

[7] E. Ganz, Whiteness: photometric specification and colorimetric evaluation, Applied Optics 15, 2039-2058 (1976).

[8] A. G. C. Puebla, Whiteness assessment: A primer, (2006).

[9] R. Griesser, Assessment of whiteness and tint of fluorescent substrates with good interinstrument correlation, Color Research and Application 19, 446-460 (1994).

[10] R. Griesser, CIE whiteness and tint : possible improvements, APPITA Journal 49, 105 (1996).

[11] David A, Krames MR, Houser KW., Whiteness metric for light sources of arbitrary color temperatures: proposal and application to light-emitting-diodes, Opt. Express, $2013 \mathrm{Jul}$ 15;21(14):16702-15. doi: 10.1364/OE.21.016702.

[12] CIE Publication 15:2004, Colorimetry, 3r d Ed ., Commission Internationale de l'E' clairage, Vienna, Austria (2004).

[13] Coppel, L.G. ; Andersson, M. ; Norberg, O. ; Lindberg, S., Impact of illumination spectral power distribution on radiance factor of fluorescing materials, Colour and Visual Computing Symposium (CVCS), 2013, doi: 10.1109/CVCS.2013.6626275

[14] Vik, M., Viková, M., Maltseva, A., Čejka, V.: Complex color non-uniformity and surface texture, 20th international conference STRUTEX, Liberec, December 1 - 2, 2014, pp. 95101, ISBN 978-80-7494-139-9

[15] M Wei, KW Houser, A David, and MR Krames: Perceptual responses to LED illumination with colour rendering indices of 85 and 97, Lighting Research and Technology 1477153514548089, first published on August 22, 2014 doi:10.1177/1477153514548089

[16] Aurelien, D., Krames, M.: High Color-Rendering, Full-VisibleSpectrum LEDs, Issue 452014 LUGER RESEARCH e.U.

Authors:

doc. Ing. Michal Vik, Ph.D., Technická univerzita v Liberci, Fakulta textilní, Laboratoř Měření Barevnosti a Vzhledu, Studentská 2, 46117 Liberec, Czech Republic, e-mail: michal.vik@tul.cz

Ing. Martina Viková, PhD., Technická univerzita v Liberci, Fakulta textilní, Laboratoř Měření Barevnosti a Vzhledu, Studentská 2, 46117 Liberec, Czech Republic, e-mail: martina.vikova@tul.cz

Aravin Prince Perisiyasamy, M.Tech., Technická univerzita v Liberci, Fakulta textilní, Laboratoř Měření Barevnosti a Vzhledu, Studentská 2, 46117 Liberec, Czech Republic,

e-mail: aravin.prince.periyasamy@tul.cz 